\title{
Transverse momentum spectra of identified charged hadrons with the ALICE detector in $\mathrm{Pb}-\mathrm{Pb}$ collisions at $\sqrt{s_{N N}}=2.76 \mathrm{TeV}$
}

\author{
Roberto Preghenella*, for the ALICE Collaboration \\ Centro Studi e Ricerche e Museo Storico della Fisica "Enrico Fermi", Rome, Italy \\ Dipartimento di Fisica dell'Università and Sezione INFN, Bologna, Italy \\ E-mail: preghenella@bo.infn.it
}

\begin{abstract}
The measurement of identified charged-hadron production at mid-rapidity $(|y|<0.5)$ performed with the ALICE experiment is presented for $\mathrm{Pb}-\mathrm{Pb}$ collisions at $\sqrt{s_{\mathrm{NN}}}=2.76 \mathrm{TeV}$. The transverse momentum spectra of $\pi^{ \pm}, K^{ \pm}, p$ and $\bar{p}$ are measured from $100 \mathrm{MeV} / \mathrm{c}$ up to $3 \mathrm{GeV} / \mathrm{c}$ for pions, from $200 \mathrm{MeV} / \mathrm{c}$ up to $2 \mathrm{GeV} / \mathrm{c}$ for kaons and from $300 \mathrm{MeV} / \mathrm{c}$ up to $3 \mathrm{GeV} / \mathrm{c}$ for protons and antiprotons using the $d E / d x$ and the time-of-flight particle-identification techniques. Preliminary results on charged hadron production yields and particle ratios are reported as a function of $p_{T}$ and collision centrality. Finally, the results are discussed in terms of hydrodynamics-inspired models and compared with published RHIC data in Au-Au collisions at $\sqrt{s_{\mathrm{NN}}}=200 \mathrm{GeV}$ and predictions for the LHC.
\end{abstract}

The 2011 Europhysics Conference on High Energy Physics, EPS-HEP 2011,

July 21-27, 2011

Grenoble, Rhône-Alpes, France

\footnotetext{
${ }^{*}$ Speaker.
} 


\section{Introduction}

ALICE (A Large Ion Collider Experiment) is a general-purpose heavy-ion experiment at the CERN LHC (Large Hadron Collider) aimed at studying the physics of strongly-interacting matter and the quark-gluon plasma. A unique design has been adopted for the ALICE detector to fulfill tracking and particle-identification requirements [1]. Thanks to these features the experiment is able to identify charged hadrons with momenta from about $0.1 \mathrm{GeV} / \mathrm{c}$ and up to a few $\mathrm{GeV} / \mathrm{c}$ by combining different detecting systems, as discussed in Section 2.

The hot and dense matter produced in ultrarelativistic heavy-ion collisions evolves through different phases to a freeze-out state where strong interactions among the hadrons stop. Since produced hadrons carry information about the evolution of the system, the measurement of the tranverse momentum distributions and yields of identified hadrons is essential to understand the global properties and dynamics of the later stages. Results on charged-hadron spectra and yields at mid-rapidity are presented in Section 3 for $\mathrm{Pb}-\mathrm{Pb}$ collisions at $\sqrt{s_{\mathrm{NN}}}=2.76 \mathrm{TeV}$.

\section{Particle identification}

In this section the particle-identification (PID) detectors relevant for this analysis are briefly discussed, namely the Inner Tracking System (ITS), the Time-Projection Chamber (TPC) and the Time-Of-Flight detector (TOF). A detailed review of the ALICE detector and of its PID capabilities can be found in [1]. The ITS is a six-layer silicon detector located at radii between 4 and $43 \mathrm{~cm}$. Four of the six layers provide $d E / d x$ measurements and are used for particle identification in the non-relativistic $\left(1 / \beta^{2}\right)$ region. Moreover, using the ITS as a standalone tracker enables one to reconstruct and identify low-momentum particles not reaching the main tracking systems. The TPC is the main central-barrel tracking detector of ALICE and provides three-dimensional hit information and specific energy-loss measurements with up to 159 samples. With the measured particle momentum and $\langle d E / d x\rangle$ the particle type can be determined by comparing the measurements against the Bethe-Bloch expectation. The TOF detector is a large-area array of Multigap Resistive Plate Chambers (MRPC) and covers the central pseudorapidity region $(|\eta|<0.9$, full azimuth). Particle identification is performed by matching momentum and trajectory-length measurements performed by the tracking system with the time-of-flight information provided by the TOF system. The total time-of-flight resolution is about $85 \mathrm{ps}$ in $\mathrm{Pb}-\mathrm{Pb}$ collisions and it is determined by the time resolution of the detector itself and by the start-time resolution.

\section{Results}

The transverse momentum spectra of primary $\pi^{ \pm}, K^{ \pm}, p$ and $\bar{p}$ are measured at mid-rapidity $(|y|<0.5)$ combining the techniques and detectors described in Section 2. Primary particles are defined as prompt particles produced in the collision and all decay products, except products from weak decay of strange particles. The contribution from the feed-down of weakly-decaying particles to $\pi^{ \pm}, p$ and $\bar{p}$ and from protons from material are subtracted by fitting the data using Monte Carlo templates of the $\mathrm{DCA}^{1}$ distributions. Hadron spectra are measured in several centrality

\footnotetext{
${ }^{1}$ Distance of Closest Approach to the reconstructed primary vertex.
} 


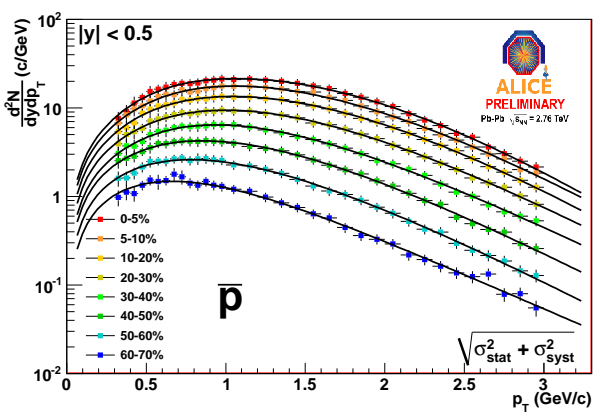

Figure 1: Transverse momentum spectra of primary $\bar{p}$ and corresponding fits in several centrality classes.

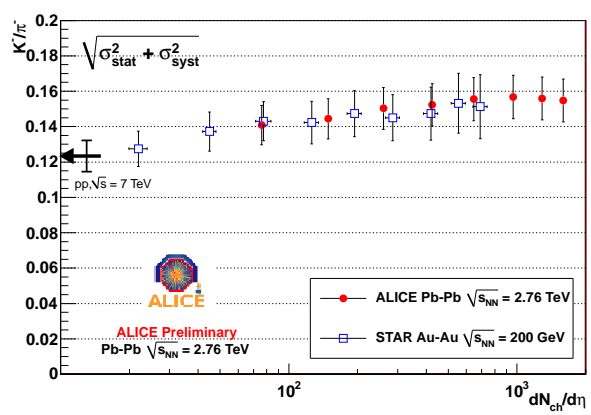

Figure 3: $K^{-} / \pi^{-}$production ratios as a function of $d N_{c h} / d \eta$ compared to RHIC data.

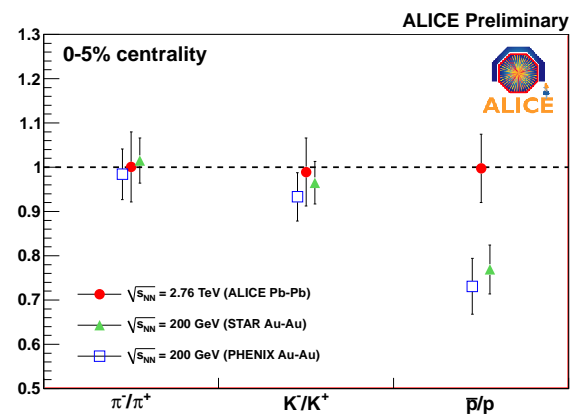

Figure 2: Antiparticle/particle production ratios in the $0-5 \%$ most central collisions.

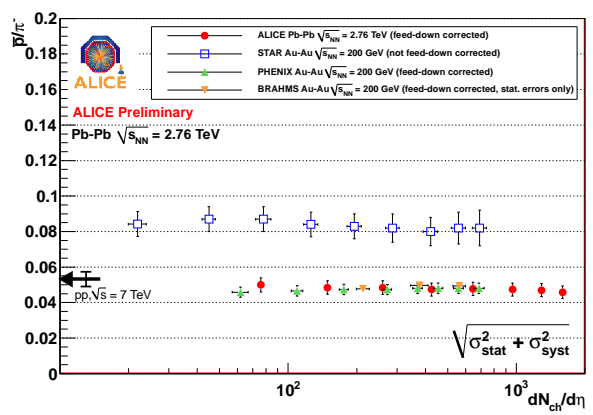

Figure 4: $\bar{p} / \pi^{-}$production ratios as a function of $d N_{c h} / d \eta$ compared to RHIC data.

classes (see [2] for details on centrality selection) from $100 \mathrm{MeV} / \mathrm{c}$ up to $3 \mathrm{GeV} / \mathrm{c}$ for pions, from $200 \mathrm{MeV} / \mathrm{c}$ up to $2 \mathrm{GeV} / \mathrm{c}$ for kaons and from $300 \mathrm{MeV} / \mathrm{c}$ up to $3 \mathrm{GeV} / \mathrm{c}$ for protons and antiprotons. Individual fits to the data are performed following a blast-wave parameterization [3] to extrapolate the spectra outside the measured $p_{T}$ range. The measured spectra and corresponding fits are shown in Figure 1 for primary $\bar{p}$. Average transverse momenta $\left\langle p_{T}\right\rangle$ and integrated production yields $d N / d y$ are obtained using the measured data points and the extrapolation.

Antiparticle/particle integrated production ratios are observed to be consistent with unity for all particle species in all centralities suggesting that the baryo-chemical potential $\mu_{B}$ is close to zero as expected at LHC energies. Figure 2 compares ALICE results with RHIC at data in $\mathrm{Au}-$ $\mathrm{Au}$ collisions at $\sqrt{s_{\mathrm{NN}}}=200 \mathrm{GeV}$ [4] for the 0-5\% most central collisions. The $p_{T}$-integrated $K^{-} / \pi^{-}$and $\bar{p} / \pi^{-}$ratios are shown in Figure 3 and 4 as a function of the charged-particle density $d N_{c h} / d \eta$ [2] and are compared with RHIC data at $\sqrt{s_{\mathrm{NN}}}=200 \mathrm{GeV}$ and ALICE proton-proton results at $\sqrt{s}=7 \mathrm{TeV}$ [5]. $K^{-} / \pi^{-}$production nicely follows the trend measured by STAR. $\bar{p} / \pi^{-}$ results are similar to previous measurements performed by PHENIX and BRAHMS where the definition of the proton sample is close to ours (proton measurements reported by STAR are inclusive). Finally, the $\bar{p} / \pi^{-}$ratio measured at the LHC $(\sim 0.05)$ is significantly lower that the value expected from statistical model predictions $(\sim 0.07-0.09)$ with a chemical freeze-out temperature of $T_{c h}=160-170 \mathrm{MeV}$ at the LHC [6].

The measured hadron $\left\langle p_{T}\right\rangle$ 's are shown in Figure 5 as a function of $d N_{c h} / d \eta$ for $\pi^{-}, K^{-}$and $\bar{p}$ and are compared to STAR results in Au-Au collisions at $\sqrt{s_{\mathrm{NN}}}=200 \mathrm{GeV}$. The spectra are observed to be harder that at RHIC for similar $d N_{c h} / d \eta$. A detailed study of the spectral shapes has 


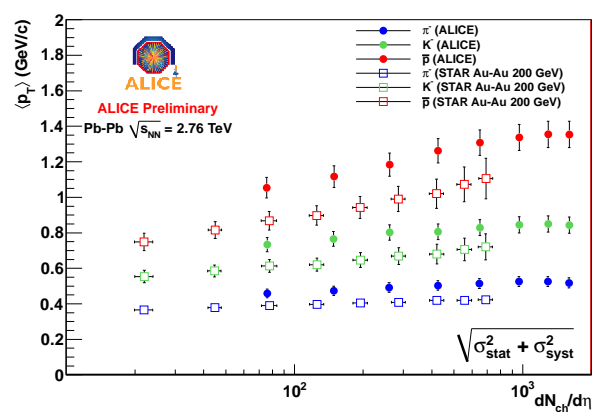

Figure 5: Hadron $\left\langle p_{T}\right\rangle$ as a function of the charged- Figure 6: Thermal freeze-out parameters $T_{f o}$ and particle density $d N_{c h} / d \eta$.

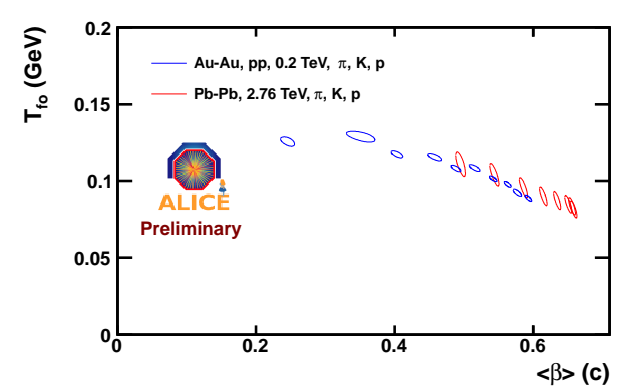

$\langle\beta\rangle$ from combined blast-wave fits.

been done in order to give a quantitative estimate of the thermal freeze-out temperature $T_{f o}$ and the average transverse flow $\langle\beta\rangle$. A combined blast-wave fit of the spectra has been performed in the ranges $0.3-1.0 \mathrm{GeV} / \mathrm{c}, 0.2-1.5 \mathrm{GeV} / \mathrm{c}$ and $0.3-3.0 \mathrm{GeV} / \mathrm{c}$ for pions, kaons and protons respectively. While the $T_{f o}$ parameter is slightly sensitive to the pion fit range because of feed-down of resonances $^{2}$ the transverse flow $\langle\beta\rangle$ measurement is not, being dominated by the proton spectral shape. The results obtained on the thermal freeze-out properties in different centrality bins are compared with similar measurements performed by the STAR Collaboration at lower energies in Figure 6. A stronger radial flow is observed with respect to RHIC, being about 10\% larger in the most central collisions at the LHC.

\section{Conclusions}

The tranverse momentum spectra of $\pi^{ \pm}, K^{ \pm}, p$ and $\bar{p}$ have been measured with ALICE in $\mathrm{Pb}$ $\mathrm{Pb}$ collisions at $\sqrt{s_{\mathrm{NN}}}=2.76 \mathrm{TeV}$, demonstrating the excellent PID capabilities of the experiment. Antiparticle/particle production ratios are consistent with unity as expected at LHC energies. $\bar{p} / \pi^{-}$ integrated ratio is significantly lower than statistical model predictions with a chemical freezeout temperature $T_{c h}=160-170 \mathrm{MeV}$. The average transverse momenta and the spectral shapes indicate a $\sim 10 \%$ stronger radial flow than at RHIC energies.

\section{References}

[1] ALICE Collaboration, J. Phys. G32, 1295 (2006)

ALICE Collaboration, J. Instrum. 3, S08002 (2008)

[2] ALICE Collaboration, Phys. Rev. Lett. 106, 032301 (2011)

[3] E. Schnedermann, J. Sollfrank and U. Heinz, Phys. Rev. C48, 2462 (1993)

[4] STAR Collaboration, Phys. Rev. C79, 034909 (2009)

PHENIX Collaboration, Phys. Rev. C69, 034909 (2004)

BRAHMS Collaboration, Phys. Rev. C72, 014908 (2005)

[5] M. Chojnacki (Quark Matter 2011 proceedings), arXiv:1109.6774v1 [hep-ex]

[6] J. Cleymans, I. Kraus, H. Oeschler, K. Redlich and S. Wheaton, Phys. Rev. C74, 034903 (2006)

A. Andronic, P. Braun-Munzinger and J. Stachel, Phys. Lett. B673, 142 (2009)

\footnotetext{
${ }^{2}$ This effect will be investigated in details in the future.
} 